\title{
Economic Evaluation of Oral Alendronate Therapy for Osteoporosis in Chinese Postmenopausal Women: The Impact of Medication Compliance and Persistence
}

\author{
Ruxu You ${ }^{1}$ and Zijie $\mathrm{Liu}^{2}$ \\ ${ }^{1}$ Department of Pharmacy, Union Hospital, Tongji Medical College, Huazhong University \\ of Science and Technology \\ ${ }^{2}$ Institute of Materia Medica, Chinese Academy of Medical Sciences and Peking Union \\ Medical College
}

June 3, 2020

\begin{abstract}
Aims: The purpose of the present research was to evaluate the cost-effectiveness of oral alendronate for individuals with osteoporosis. We also assessed the impact of medication compliance and persistence on economic outcomes of alendronate, and potential economic evaluations of persistence-enhancing interventions. Methods: We constructed an individual-level statetransition model to project health outcomes and costs of oral alendronate for Chinese postmenopausal osteoporotic women. The impact of medication compliance and persistence on economic evaluation was addressed in various scenario analyses. Model inputs were derived from clinical trials and published sources where available. Deterministic and probabilistic sensitivity analyses were conducted to explore the impact of uncertainties and assumptions on the cost-effectiveness results. Results: Compared to no treatment, alendronate treatment was associated with an additional 0.052 QALYs at an additional cost of USD 738, which yielded an ICER of USD 14,192.308/QALY. The ICER for the different scenarios (full compliance, full persistence, both full persistence and full compliance) were USD 4933.333/QALY, USD 3006.84/QALY and USD 2019.822/QALY, respectively. One-way sensitivity analysis showed the ICER was most sensitive to variations in time horizon and residual effect. Probabilistic sensitivity analysis demonstrated that, at a willingness-to-pay of USD 29,340/QALY, the probability that oral alendronate therapy will be cost-effective is approximate $80 \%$. Conclusions: The findings support the view that oral alendronate is costeffective for the treatment of osteoporotic fractures in Chinese postmenopausal women. Medication persistence is found to have a greater impact on cost-effectiveness than compliance, and interventions to improve persistence to be an efficient use of resources.
\end{abstract}

\section{What is already known about this subject}

Poor medication compliance and persistence are common problems of osteoporosis management and they affect both the clinical and cost-effectiveness of osteoporosis interventions. The potential loss of benefits resulting from poor compliance and persistence with oral alendronate in the China setting has not been well described.

\section{What this study adds}

- This study explored the cost-effectiveness of oral alendronate therapy for postmenopausal osteoporotic women from the perspective of Chinese health care payer.

- We incorporated medication persistence and compliance into our hybrid modeling and extensively examined how these changes in parameters have an impact on the pharmacoeconomic evaluation of oral alendronate treatment. 
- Medication persistence is found to have a greater impact on cost-effectiveness than compliance, and we further assessed the potential economic value of persistence-enhancing interventions according to a given range of their costs and effectiveness values.

\section{Introduction}

Osteoporosis, or porous bone, is a disease characterized by low bone mass and structural deterioration of bone tissues, leading to bone fragility and an increased risk of fractures ${ }^{1}$. The International Osteoporosis Foundation (IOF) estimates that by 2050, more than $50 \%$ of all osteoporotic fractures will occur in Asia-and China will be most severely affected due to its large population of seniors ${ }^{2}$. Fractures significantly affect patients by impairing their ability to perform daily activities. Moreover, a health economics model was developed and forecasted that the costs of osteoporotic fractures in China will double by 2035, and rising to approximately USD 25.58 billion by 2050, indicating that in addition to morbidity and mortality, osteoporotic fractures are also associated with a significant health care expenditure to the society ${ }^{3,4}$.

Fortunately, medical advancements have increased the range of therapeutic options available for the prevention and treatment of fractures ${ }^{5}$. Currently, oral bisphosphonates are the most potent antiresorptive drugs for treatment of osteoporosis in postmenopausal women ${ }^{6}$. Multiple meta-analyses and systematic reviews have shown that bisphosphonates are effective in decreasing the risk of various types of bone fractures ${ }^{7,8}$. However, it is widely acknowledged that compliance and persistence with oral osteoporosis medications is poor $^{9-11}$. A recent observational study estimated that $53 \%$ of the study population achieved a medication possession ratio (MPR) of $80 \%$ or higher 6 months after initiating therapy, and the equivalent value for 7-12 months was only $43 \%^{12}$. Persistence, or the length of time a patient continues therapy, is similarly poor. It has been reported that the rate of persistence among new users was $46 \%$ after 7-12 months treatment period ${ }^{12}$.

Although poor compliance and persistence decrease the cost of the intervention, the effectiveness of treatment is also reduced, which reduces bone mineral density, and in turn leads to higher risk of fractures ${ }^{13,14}$. Hence, in order to estimate the cost-effectiveness of the intervention in real-world settings, it is important that economic evaluations take compliance and persistence into account.

The purpose of the present research was to evaluate the potential loss of benefits resulting from poor compliance and persistence with oral alendronate in osteoporotic individuals. More specifically, we first compared the clinical and economic outcomes derived from real-life setting with those expected with full compliance and persistence. In additional, we further evaluated the potential economic value of persistence-enhancing interventions.

\section{Methods}

\subsection{Overview}

The development of this model adhered to the recommendations for the conduct of economic evaluations in osteoporosis ${ }^{15}$. We used an updated version of previously validated individual-level state-transition model ${ }^{16}$ to estimate the impact of the compliance and persistence on the cost effectiveness of alendronate treatment for Chinese postmenopausal osteoporotic women aged 65 and older. The model estimated the outcomes including number of fractures quality adjusted life-years (QALYs); direct societal costs in 2018 US dollars (USD); and incremental cost-effectiveness ratios (ICERs) per QALY gained. Costs and health outcomes beyond the first year were discounted at an annual of $3 \%$, which is consistent with Chinese guidelines for pharmacoeconomic evaluations ${ }^{17}$. We assessed cost-effectiveness from the health care payer perspectives and considered three times of per capita gross domestic product of China in 2018 ( USD 29,340) as the willingness-to-pay (WTP) threshold. We used TreeAge Pro 2018 (TreeAge Software Inc., Williamston, MA, USA) to perform our analyses.

\subsection{Model structure}

We modeled the disease progression of osteoporosis through six states: no fracture, hip fracture, clinical 
vertebral fracture, wrist fracture, other osteoporotic fracture, and death. The other osteoporotic fracture state as defined by the IOF-EFPIA report ${ }^{18}$. The cycle length of the model was 1 year which chosen to represent a clinically meaningful time interval. Each individual can sustain only one fracture per cycle, and can experience up to two hip fractures but unlimited clinical vertebral, wrist and other osteoporotic fractures during the entire study period. We used tracker variables to record individual characteristics and disease histories, which adjusted transition probabilities, costs and utilities. Table $\mathbf{1}$ shows the key parameters used in the health economics model. A more detailed description of the model can be found in our previously published work ${ }^{16}$.

\subsection{Fracture incidence and mortality rates}

Hip and vertebral fracture incidences were derived from reported epidemiological data in China ${ }^{19,}{ }^{20}$. Estimation of the incidence rates of the wrist and other osteoporotic fractures in the Chinese context was not available, hence we utilized data collected from an Asian population ${ }^{21,22}$. The incidence of fracture in the general population was further adjusted to accurately reflect the fracture risks of women with osteoporosis. The method calculated the relative risks for bone mineral density using a method previously described 23-25.

Baseline mortality rates for age-stratified Chinese women were retrieved from the China Public Health Statistical Yearbook ${ }^{26}$ and an increased mortality was assumed for individuals who experienced the hip fracture ${ }^{27}$. Because excess mortality may be attributable to comorbidities in this older population, only $25 \%$ of the excess mortality was considered to be attributable to the fractures themselves ${ }^{28}$. There was no increase in mortality following clinical vertebral, wrist and other fractures ${ }^{29,} 30$.

\subsection{Treatment}

We assumed that treated women received alendronate $70 \mathrm{mg}$ once weekly for five years. Relative risks for fractures in women taking alendronate were based on the recent systematic reviews ${ }^{31,32}$. It was assumed that reductions in fracture risk during therapy were consistent regardless of patients' age and there was no significant change in bioequivalence between brand name and generic drugs. We also assigned the cost of one general consultation visit, bone mineral density and biochemical test per year, as suggested by the Chinese guidelines for the diagnosis and treatment of primary osteoporosis ${ }^{33}$.

Inadequate medication compliance and persistence are known to be major problems in all patients with osteoporotic disease ${ }^{34}$. We considered compliance and persistence rates of alendronate obtained on the observational studies in the Chinese or Asian population ${ }^{35,36}$. Compliance rates with oral alendronate were higher in clinical than observational studies. The influence of their difference was incorporated into the microsimulation model by assuming a linear relationship between the relative risk reduction and medication compliance ${ }^{29,30}$. In addition, we modeled the residual effects of alendronate for those who discontinue therapy (called offset-time effect). We assumed that if individuals stopped treatment, they received no further therapy and offset-time was assumed to be equal to their treatment period ${ }^{11}$.

\subsection{Costs}

The cost of alendronate was based on different brand prices and corresponding market share in China. Total medication costs were multiplied by their compliance and persistence level. We charged the cost of 6month alendronate supply for individuals who discontinued alendronate within the first year. The estimated annual costs related to hip fracture of the first year and long-term care costs were obtained from previously published studies in Chinese setting ${ }^{37}, 38$. Costs of physician visits, DXA scan, laboratory tests and nursing home residence were collected from the health system or the National Development and Reform Commission of China ${ }^{39}$. All original costs were converted to a common currency and price year, 2018 United States dollars (USD), given the latest version of a web-based cost converter ${ }^{40}$.

\subsection{Utilities}

The Chinese National Health Services Survey in China has established the utility values in osteoporosis ${ }^{41}$. 
No disutilities was assumed for simulated individuals without fractures. Fracture events were associated with decrements in utility values which differed between the fracture sites and time. The quality of life multipliers were based on a recent meta-analysis ${ }^{42,} 43$.

\subsection{Model simulation and sensitivity analysis}

We performed base-case, deterministic (one-way) sensitivity, probabilistic sensitivity and scenario analyses. For baseline analysis, we ran the model with 100,000 iterations (100,000 individuals through the model one at a time). One-way sensitivity analysis was undertaken to examine the effect of each key model parameter, including fracture costs and disutilities, medication costs, initial age of treatment, time horizon, residual effect and discount rates. Probabilistic sensitivity analysis was conducted to evaluate the impact of the joint uncertainty surrounding the model variables using Monte-Carlo simulations (1000 simulations and 10,000 trials per simulation). We also examined different scenarios in which: (A) the individuals with full compliance, (B) the individuals with full persistence, (C) the individuals with both full persistence and full compliance, and (D) potential persistence-enhancing interventions.

\section{Results}

\subsection{Model validation}

The probability of dying by 105 years for untreated individuals at the ages of $65,70,75,80$ predicted by our model were $99.0 \%, 98.8 \%, 98.5 \%$ and $98.5 \%$, respectively. Model-predicted mortality risks were comparable to the Chinese life table ${ }^{26}$. We also projected that without an intervention, the cumulative probability of having at least one hip fracture or clinical vertebral fracture equal to $11.099 \%$ and $39.693 \%$, respectively, which is comparable to the epidemiological data in China ${ }^{33}$.

\subsection{Base-case findings}

Table 2 presented the total health care costs, number of fractures, QALYs and ICER estimated by the model. Compared with no treatment (mean cost USD 9411; mean effect 12.623 QALYs), alendronate treatment in the real-world setting (mean cost USD 10,149; mean effect 12.675 QALYs) was associated with overall increase in total health care cost of USD 738 and in QALYs of 0.052 , yielded in an ICER of USD 14,192.308/QALY gained. Besides, both NMB and NHB were positive, further indicated oral alendronate is more cost-effective than no intervention.

\subsection{Sensitivity analyses findings}

Deterministic sensitivity analysis showed that the most impactful parameters in the model were the time horizon and the residual effect. The ICER was markedly increased to USD 994,000/QALY when reducing the time horizon from lifetime to 5 years. Assuming no residual effect following treatment resulted in the ICER increased to USD 49,294.118/QALY (Table 3 ).

Probabilistic sensitivity analysis confirmed the aforementioned results (Figure $\mathbf{1}$ ). At a willingness-to-pay threshold of USD 29,340/QALY, the probability that alendronate would be cost-effective was approximate $80 \%$ for individuals age 65 .

\subsection{Scenario analyses findings}

The results of the scenario analysis considering alendronate therapy compliance and persistence were shown in Table 2 andFigure 2 . The lifetime cost per person was USD 9707 for the full compliance scenario, USD 9850 for the full persistence scenario, and USD 9987 for both full persistence and full compliance scenario. Total cost was lower in the scenario analysis than in the real-world setting, as the prevented costs of treating additional osteoporotic fractures resulting from non-compliance and persistence exceed the cost of the additional therapy induced by the improved compliance and persistence.

Effectiveness was measured as the number of all osteoporotic fractures and quality-adjusted life-years. The lifetime number of all fractures per person was 1.438 for the full compliance scenario, 1.418 for the full persistence, and 1.350 for both full compliance and full persistence. Hence, the number of osteoporotic 
fractures prevented in real-world setting represent $81.2 \%, 43.8 \%$, and $17.1 \%$ to that estimated with full compliance, full persistence, and both full compliance and full persistence scenario, respectively. Mean lifetime QALYs were estimated at 12.683, 12.769, and 12.904 in all scenarios tested, respectively. The QALYs gained in the real-world scenario represents $86.7 \%, 35.6 \%$, and $18.5 \%$ to that obtained under the above three scenarios, respectively.

Compared to no treatment, the ICER for the three scenarios ranged from USD 2019.822/QALY to USD 4933.333/QALY. These results were all lower than that derived from real-world analysis. It should be noted that three different scenarios were associated with lower costs and great QALYs than the real-world setting, indicated that the improvement of compliance and persistence was found to be cost-saving.

Figure 3 displayed the economic assessment of persistence-enhancing interventions based on differential reduction in treatment discontinuation and their corresponding cost. When the reductions in treatment discontinuation were high $(>30 \%)$ and the invention costs were low ( $<$ USD 100), the ICER was less than USD 9780/QALY (1× GDP per capita) and could be considered highly cost-effective. Conversely, when the invention costs were high (> USD 400) and the reductions in treatment discontinuation were low $(<10 \%)$, the ICER was more than USD 29340/QALY $(3 \times$ GDP per capita $)$ and could be considered not cost-effective. For other potential combination of values within the given range, the ICER between USD 9780/QALY and USD 29,340/QALY, which regarded as acceptable cost-effectiveness limits.

\section{Discussions}

In this study, we used a modeling approach incorporating the medication compliance and persistence to examine the cost-effectiveness of oral alendronate treatment versus no intervention in the treatment of osteoporosis in Chinese postmenopausal women. Our base case analysis revealed that compared with no treatment, oral alendronate therapy $70 \mathrm{mg}$ once weekly for five years was a high-value treatment at a willingness-to-pay threshold of USD 29,340/QALY.

The key variable in the current research was the medication persistence and compliance. Although oral alendronates have been demonstrated to be high value with current medication discipline, they are more cost-effective with full compliance and persistence. In addition, persistence was found to have a greater impact on cost-effectiveness than compliance. Full persistence in our model would yield an ICER of USD 3006.849/QALY, lower than the equivalent value for the full compliance (USD 4933.333/QALY). It should be noted that this heightened persistence rate of oral alendronate was emphasized by our assumption of a residual effect from treatment; the risk for fracture returned to rates in the absence of therapy over the same years as the treatment duration in a gradual linear fashion after completing the therapy. This is also examined by deterministic sensitivity analyses, in which we assumed no residual effect after the treatment, the ICER of oral alendronate was sharply increased to USD 9294.118/QALY. Hence, interventions to enhance persistence are necessary to decrease the considerable economic burden caused by the non-persistence with oral alendronate.

Our results confirmed prior work that it is important to include medication persistence and compliance in pharmacoeconomic analysis of osteoporosis treatment. The two studies of Hiligsmann and colleagues ${ }^{13,} 14$ which were focused on oral bisphosphonates suggested that poor adherence with osteoporosis medications results in approximately a $50 \%$ reduction in the potential benefits observed in clinical trials and a doubling of the cost per QALY gained from these medications. Programs to improve compliance were considered to be an efficient use of resources. In contrast, the study of Chen and colleagues' 37 in the China setting compared raloxifene treatment with conventional treatment (Alendronate, Calcitonin, Calcium combined with vitamin D) found opposite results. In this study, although high persistence and compliance increased both clinical effectiveness and average costs, the improvement on effectiveness was marginal in their research, thus resulting in higher ICER compared with the real-world scenario. The main reasons for such a difference could be attributed to the costs for fracture inpatients and the comparator.

In our previous study ${ }^{16}$, in which we examined cost-effective of once-yearly injection of zoledronic acid compared with oral alendronate once weekly for postmenopausal osteoporotic women without prior history 
of fracture in China, we concluded that zoledronic acid was cost-effective at all starting ages and even costsaving in scenario analysis mainly based on zoledronic acid's higher persistence leading to higher efficacy. In this study, we came to a similar conclusion that the medication persistence plays a key role in shaping perceptions of fracture risk and osteoporosis drug effectiveness. In addition, we extend the prior work by design a meaningful framework for assessing the economic value of persistence-enhancing interventions. We assessed the potential combination of the intervention costs from USD 100-500 and the relative reduction in discontinuation from $10 \%-50 \%$.

There are limitations associated with the current study. First, like all models, generalizability of the results to the target population of other races/ethnicities or in other countries may be uncertain due to the heterogeneity of payer perspectives and the country-specific epidemiologic data used. Moreover, although much of the data constructed the model were obtained from Chinese context, some data were also extrapolated from other countries. An updated pharmacoeconomic analysis should be explored when these data are available in Chinese setting. Second, compliance and persistence rates were derived from a retrospective study ${ }^{35}$ in which whether patients actually took the dispensed drug is unknown. The study assumed that patients who obtain prescription refills do take their medications based on chart review. As a result, compliance may be overestimated. Third, our analysis did not examine the impact of restart therapy after discontinuation. We assumed those who did not take alendronate continued not to take medication in this model, which may not always mimic treatment in the real world because some patients might return to treatment after this period. Finally, we did not perform a budget impact analysis to assess the potential cost savings of this strategy. Due to the enormous amount of osteoporosis cases in China, the financial burdens for the health care system might be heavy.

Despite these limitations, our research has several key strengths. First, to the best of our knowledge, this is the first pharmacoeconomic analysis that compared oral alendronate to no treatment in a Chinese population. Second, we incorporated medication persistence and compliance, which are considered to be critical impede to osteoporosis management, into our hybrid modeling and extensively examined how these changes in parameters have an impact on model results. We further assessed the potential cost-effectiveness of persistence-enhancing interventions according to a given range of their costs and effectiveness values.

\section{Conclusion}

In conclusion, oral alendronate is considered to be a high-value therapy option for postmenopausal osteoporotic women from the perspective of Chinese health care payer, and further interventions to improve osteoporosis medication persistence will likely have favorable ICERs.

\section{Acknowledgment}

The authors acknowledge Takahiro Mori for the contribution to build the Markov model, which we updated and used in our analysis.

\section{Conflict of interest statement}

There are no competing interests to declare.

\section{Funding information}

This research did not receive any specific grant from any funding agency in the public, commercial or notfor-profit sector.

\section{Contributors}

ZJ Liu conceived and designed the research, RX You collected and analyzed the data. All authors wrote the manuscript.

\section{References}


[1] Watts N B. Postmenopausal Osteoporosis: A Clinical Review. J Womens Health (Larchmt). 2018; 27(9): 1093-1096.

[2] Pisani P, Renna M D, Conversano F, et al. Major osteoporotic fragility fractures: Risk factor updates and societal impact. World journal of orthopedics. 2016; 7(3): 171-181.

[3] Si L, Winzenberg T M, Jiang Q, et al. Projection of osteoporosis-related fractures and costs in China: 2010-2050. Osteoporos Int. 2015; 26(7): 1929-1937.

[4] Liu R, Chao A, Wang K, et al. Incidence and risk factors of medical complications and direct medical costs after osteoporotic fracture among patients in China. Archives of Osteoporosis. 2018; 13(1): 12.

[5] Iolascon G, Moretti A, Toro G, et al. Pharmacological Therapy of Osteoporosis: What's New? Clinical interventions in aging. 2020; 15: 485-491.

[6] Maraka S, Kennel K A. Bisphosphonates for the prevention and treatment of osteoporosis. BMJ: British Medical Journal. 2015; 351: h3783.

[7] Feng Z, Zeng S, Wang Y, et al. Bisphosphonates for the prevention and treatment of osteoporosis in patients with rheumatic diseases: a systematic review and meta-analysis. PloS one. 2013; 8(12): e80890.

[8] Brown J P, Morin S, Leslie W, et al. Bisphosphonates for treatment of osteoporosis: expected benefits, potential harms, and drug holidays. Canadian family physician Medecin de famille canadien. 2014; 60(4): 324-333.

[9] Imaz I, Zegarra P, Gonzalez-Enriquez J, et al. Poor bisphosphonate adherence for treatment of osteoporosis increases fracture risk: systematic review and meta-analysis. Osteoporos Int. 2010; 21(11): 1943-1951.

[10] Fatoye F, Smith P, Gebrye T, et al. Real-world persistence and adherence with oral bisphosphonates for osteoporosis: a systematic review. BMJ open. 2019; 9(4): e27049.

[11] Hiligsmann M, Boonen A, Rabenda V, et al. The importance of integrating medication adherence into pharmacoeconomic analyses: the example of osteoporosis. Expert Rev Pharmacoecon Outcomes Res. 2012; 12(2): 159-166.

[12] Kothawala P, Badamgarav E, Ryu S, et al. Systematic review and meta-analysis of real-world adherence to drug therapy for osteoporosis. Mayo Clin Proc. 2007; 82(12): 1493-1501.

[13] Hiligsmann M, Mcgowan B, Bennett K, et al. The clinical and economic burden of poor adherence and persistence with osteoporosis medications in Ireland. Value Health. 2012; 15(5): 604-612.

[14] Hiligsmann M, Rabenda V, Bruyere O, et al. The clinical and economic burden of non-adherence with oral bisphosphonates in osteoporotic patients. Health Policy. 2010; 96(2): 170-177.

[15] Hiligsmann M, Reginster J Y, Tosteson A, et al. Recommendations for the conduct of economic evaluations in osteoporosis: outcomes of an experts' consensus meeting organized by the European Society for Clinical and Economic Aspects of Osteoporosis, Osteoarthritis and Musculoskeletal Diseases (ESCEO) and the US branch of the International Osteoporosis Foundation. Osteoporos Int. 2019; 30(1): 45-57.

[16] You R, Zhang Y, Wu D B, et al. Cost-Effectiveness of Zoledronic Acid Versus Oral Alendronate for Postmenopausal Osteoporotic Women in China. Frontiers in Pharmacology. 2020; 11: 456.

[17] Liu G. China Guidelines for Pharmacoeconomic Evaluations. 1st ed. Beijing: Sciencepress, 2011.

[18] Svedbom A, Hernlund E, Ivergård M, et al. Osteoporosis in the European Union: a compendium of country-specific reports. Archives of Osteoporosis. 2013; 8(1): 137.

[19] Bow C H, Cheung E, Cheung C L, et al. Ethnic difference of clinical vertebral fracture risk. Osteoporos Int. 2012; 23(3): 879-885. 
[20] Wang J, Wang Y, Liu W D, et al. Hip fractures in Hefei, China: the Hefei osteoporosis project. J Bone Miner Metab. 2014; 32(2): 206-214.

[21] Lofthus C M, Frihagen F, Meyer H E, et al. Epidemiology of distal forearm fractures in Oslo, Norway. Osteoporos Int. 2008; 19(6): 781-786.

[22] Melton Iii L J, Crowson C S, O Fallon W M. Fracture Incidence in Olmsted County, Minnesota: Comparison of Urban with Rural Rates and Changes in Urban Rates Over time. Osteoporosis International. 1999; 9(1): 29-37.

[23] Kanis J A, Johnell O, Oden A, et al. Risk of hip fracture according to the World Health Organization criteria for osteopenia and osteoporosis. Bone. 2000; 27(5): 585-590.

[24] Johnell O, Kanis J A, Oden A, et al. Predictive value of BMD for hip and other fractures. J Bone Miner Res. 2005; 20(7): 1185-1194.

[25] Marshall D, Johnell O, Wedel H. Meta-analysis of how well measures of bone mineral density predict occurrence of osteoporotic fractures. BMJ (Clinical research ed.). 1996; 312(7041): 1254-1259.

[26] National Health Committee of the People's Republic of China. China health statistical yearbook. Beijing: Peking Union Medical College Publishing House, 2018.

[27] Haentjens P, Magaziner J, Colón-Emeric C S, et al. Meta-analysis: excess mortality after hip fracture among older women and men. Annals of internal medicine. 2010; 152(6): 380-390.

[28] Kanis J A, Oden A, Johnell O, et al. The components of excess mortality after hip fracture. Bone. 2003; $32(5): 468-473$.

[29] Mori T, Crandall C J, Ganz D A. Cost-effectiveness of denosumab versus oral alendronate for elderly osteoporotic women in Japan. Osteoporos Int. 2017; 28(5): 1733-1744.

[30] Mori T, Crandall C J, Ganz D A. Cost-effectiveness of combined oral bisphosphonate therapy and falls prevention exercise for fracture prevention in the USA. Osteoporos Int. 2017; 28(2): 585-595.

[31] Murad M H, Drake M T, Mullan R J, et al. Clinical review. Comparative effectiveness of drug treatments to prevent fragility fractures: a systematic review and network meta-analysis. J Clin Endocrinol Metab. 2012; 97(6): 1871-1880.

[32] Wells G, Cranney A, Peterson J, et al. Risedronate for the primary and secondary prevention of osteoporotic fractures in postmenopausal women. Cochrane Database Syst Rev. 2008(1): D4523.

[33] Chinese Society of Osteoporosis and Bone Mineral Research. Guidelines for the diagnosis and management of primary osteoporosis. Chin J Osteoporos. 2019; 25(3): 281-309.

[34] Stevenson M D, Selby P L. Modelling the Cost Effectiveness of Interventions for Osteoporosis: Issues to Consider. PharmacoEconomics. 2014; 32(8): 735-743.

[35] Cheng T, Yu S, Hsu C, et al. Differences in Adherence to Osteoporosis Regimens: A 2-Year Analysis of a Population Treated Under Specific Guidelines. Clinical Therapeutics. 2013; 35(7): 1005-1015.

[36] Kishimoto H, Maehara M. Compliance and persistence with daily, weekly, and monthly bisphosphonates for osteoporosis in Japan: analysis of data from the CISA. Archives of osteoporosis. 2015; 10: 231.

[37] Si L, Winzenberg T M, Chen M, et al. Screening for osteoporosis in Chinese post-menopausal women: a health economic modelling study. Osteoporosis International. 2016; 27(7): 2259-2269.

[38] Qu B, Ma Y, Yan M, et al. The economic burden of fracture patients with osteoporosis in western China. Osteoporos Int. 2014; 25(7): 1853-1860.

[39] National Development and Reform Commission. [Online]. Available: http://www.ndrc.gov.cn[Accessed September 20 2018]. 
[40] CCEMG-EPPI-Centre Cost Converter Available. [Online]. Available: http://eppi.ioe.ac.uk/costconversion/default.aspx [Accessed September 20 2018].

[41] Sun S, Chen J, Johannesson M, et al. Population health status in China: EQ-5D results, by age, sex and socio-economic status, from the National Health Services Survey 2008. Qual Life Res. 2011; 20(3): 309-320.

[42] Si L, Winzenberg T M, de Graaff B, et al. A systematic review and meta-analysis of utility-based quality of life for osteoporosis-related conditions. Osteoporos Int. 2014; 25(8): 1987-1997.

[43] Hiligsmann M, Ethgen O, Richy F, et al. Utility values associated with osteoporotic fracture: a systematic review of the literature. Calcif Tissue Int. 2008; 82(4): 288-292.

Table 1 Summary of key parameters in the model

\begin{tabular}{|c|c|c|}
\hline Parameter & Value & Range \\
\hline \multicolumn{3}{|l|}{ Alendronate therapy } \\
\hline Relative risk of hip fracture & 0.45 & $0.27-0.68$ \\
\hline Relative risk of clinical vertebral fracture & 0.50 & $0.33-0.79$ \\
\hline Relative risk of wrist fracture & 0.50 & $0.34-0.73$ \\
\hline Relative risk of other fracture & 0.78 & $0.66-0.92$ \\
\hline Persistence rate & 0.57 (year 1) & $\mathrm{N} / \mathrm{A}$ \\
\hline Compliance rate & $0.71($ year 1$)$ & $\mathrm{N} / \mathrm{A}$ \\
\hline \multicolumn{3}{|l|}{ Costs (2018 US dollars) } \\
\hline Annual cost for Alendronate & 761.64 & $533.15-990.13$ \\
\hline Hip fracture, direct costs & 7103.25 & $4972.28-9234.23$ \\
\hline Clinical vertebral fracture, direct costs & 1310.11 & $917.08-1703.14$ \\
\hline Wrist fracture, direct costs & 967.34 & $677.14-1257.54$ \\
\hline Other fracture, direct costs & 1692.41 & $1184.69-2200.13$ \\
\hline Annual cost for the post-hip fracture & 4438.08 & $3106.66-5769.50$ \\
\hline DXA scan & 85 & $59.5-110.5$ \\
\hline Blood tests & 72 & $50.4-93.6$ \\
\hline Physician visit & 10 & $7-13$ \\
\hline \multicolumn{3}{|l|}{ Utilities } \\
\hline Age $65-69$ & 0.806 & $0.765-0.846$ \\
\hline Age $70-74$ & 0.747 & $0.709-0.784$ \\
\hline Age $75-79$ & 0.731 & $0.694-0.767$ \\
\hline Age $80-84$ & 0.699 & $0.664-0.733$ \\
\hline Age $85+$ & 0.676 & $0.642-0.709$ \\
\hline Hip fracture, first year(multiplier) & 0.776 & $0.720-0.844$ \\
\hline Hip fracture, subsequent year(multiplier) & 0.855 & $0.800-0.909$ \\
\hline Clinical vertebral fracture, first year(multiplier) & 0.724 & $0.667-0.779$ \\
\hline Clinical vertebral fracture, subsequent year(multiplier) & 0.868 & $0.827-0.922$ \\
\hline Wrist fracture(multiplier) & 0.940 & $0.910-0.960$ \\
\hline Other fracture(multiplier) & 0.910 & $0.880-0.940$ \\
\hline \multicolumn{3}{|c|}{ Annual fracture incidence per 1000 persons (without intervention) } \\
\hline Hip fracture, age $65-69$ & 0.96 & $\mathrm{~N} / \mathrm{A}$ \\
\hline Hip fracture, age $70-74$ & 2.33 & $\mathrm{~N} / \mathrm{A}$ \\
\hline Hip fracture, age $75-79$ & 4.08 & $\mathrm{~N} / \mathrm{A}$ \\
\hline Hip fracture, age $80-84$ & 6.44 & $\mathrm{~N} / \mathrm{A}$ \\
\hline Hip fracture, age $85+$ & 6.59 & $\mathrm{~N} / \mathrm{A}$ \\
\hline Clinical vertebral fracture, age $65-69$ & 5.64 & $\mathrm{~N} / \mathrm{A}$ \\
\hline Clinical vertebral fracture, age $70-74$ & 8.74 & $\mathrm{~N} / \mathrm{A}$ \\
\hline Clinical vertebral fracture, age $75-79$ & 12.05 & $\mathrm{~N} / \mathrm{A}$ \\
\hline
\end{tabular}




\begin{tabular}{|c|c|c|}
\hline Parameter & Value & Range \\
\hline Clinical vertebral fracture, age $80-84$ & 21.19 & $\mathrm{~N} / \mathrm{A}$ \\
\hline Clinical vertebral fracture, age $85+$ & 26.89 & $\mathrm{~N} / \mathrm{A}$ \\
\hline Wrist fracture, age $65-69$ & 12.95 & $\mathrm{~N} / \mathrm{A}$ \\
\hline Wrist fracture, age $70-74$ & 13.17 & $\mathrm{~N} / \mathrm{A}$ \\
\hline Wrist fracture, age $75-79$ & 13.87 & $\mathrm{~N} / \mathrm{A}$ \\
\hline Wrist fracture, age $80-84$ & 15.01 & $\mathrm{~N} / \mathrm{A}$ \\
\hline Wrist fracture, age $85+$ & 15.10 & $\mathrm{~N} / \mathrm{A}$ \\
\hline Other osteoporotic fracture, age 65-69 & 6.60 & $\mathrm{~N} / \mathrm{A}$ \\
\hline Other osteoporotic fracture, age $70-74$ & 9.84 & $\mathrm{~N} / \mathrm{A}$ \\
\hline Other osteoporotic fracture, age 75-79 & 14.44 & $\mathrm{~N} / \mathrm{A}$ \\
\hline Other osteoporotic fracture, age $80-84$ & 18.06 & $\mathrm{~N} / \mathrm{A}$ \\
\hline Other osteoporotic fracture, age $85+$ & 26.06 & $\mathrm{~N} / \mathrm{A}$ \\
\hline \multicolumn{3}{|l|}{ Relative risks of fractures for individuals with osteoporosis } \\
\hline Hip fracture, age $65-69$ & 3.91 & $3.28-4.56$ \\
\hline Hip fracture, age $70-74$ & 3.13 & $2.80-3.47$ \\
\hline Hip fracture, age $75-79$ & 2.60 & $2.39-2.82$ \\
\hline Hip fracture, age $80-84$ & 2.04 & $1.91-2.17$ \\
\hline Hip fracture, age $85+$ & 1.92 & $1.78-2.05$ \\
\hline Clinical vertebral fracture, age $65-69$ & 2.59 & $1.19-4.27$ \\
\hline Clinical vertebral fracture, age 70-79 & 2.15 & $1.15-3.15$ \\
\hline Clinical vertebral fracture, age $80+$ & 1.82 & $1.12-2.41$ \\
\hline Wrist fracture, age $65-69$ & 1.78 & $1.78-2.19$ \\
\hline Wrist fracture, age 70-79 & 1.6 & $1.60-1.88$ \\
\hline Wrist fracture, age $80+$ & 1.45 & $1.45-1.64$ \\
\hline Other osteoporotic fracture, age $65-69$ & 2.19 & $1.78-2.59$ \\
\hline Other osteoporotic fracture, age 70-79 & 1.88 & $1.60-2.15$ \\
\hline Other osteoporotic fracture, age $80+$ & 1.64 & $1.45-1.82$ \\
\hline \multicolumn{3}{|l|}{ Annual mortality rate } \\
\hline $65-69$ & 0.01031 & $\mathrm{~N} / \mathrm{A}$ \\
\hline $70-74$ & 0.02036 & $\mathrm{~N} / \mathrm{A}$ \\
\hline 75-79 & 0.03784 & $\mathrm{~N} / \mathrm{A}$ \\
\hline $80-84$ & 0.06998 & $\mathrm{~N} / \mathrm{A}$ \\
\hline $85+$ & 0.13603 & $\mathrm{~N} / \mathrm{A}$ \\
\hline \multicolumn{3}{|l|}{ Excess mortality after a hip fracture } \\
\hline Relative hazard for mortality within a year after a hip fracture & 2.87 & $2.52-3.27$ \\
\hline Relative hazard for mortality for second and beyond after a hip fracture & 1.73 & $1.56-1.90$ \\
\hline $\begin{array}{l}\text { Proportion of excess mortality after a hip fracture directly attributable to a hip fracture } \\
\text { Discounts }\end{array}$ & 0.25 & $\mathrm{~N} / \mathrm{A}$ \\
\hline \multicolumn{3}{|l|}{ Discounts } \\
\hline Costs & 0.03 & $0-0.05$ \\
\hline Effectiveness & 0.03 & $0-0.05$ \\
\hline
\end{tabular}

Table 2 Results of base case and scenario analyses

\begin{tabular}{lllll}
\hline & Different scenarios & Different scenarios & Different scenarios & Different \\
\hline & NT & RW & FC & FP \\
Patient cost over lifetime (2018 USD) & & & 1021 & 1943 \\
Treatment cost & 0 & 890 & 8670 & 7907 \\
Total disease cost & 9411 & 3712 & 3610 & 3427 \\
Acute fracture cost & 3768 & &
\end{tabular}




\begin{tabular}{lllll}
\hline & Different scenarios & Different scenarios & Different scenarios & Different \\
\hline $\begin{array}{l}\text { Long-term fracture cost } \\
\text { Total health care cost }\end{array}$ & 5643 & 5542 & 5060 & 4480 \\
$\begin{array}{l}\text { Outcome over lifetime } \\
\text { All fractures per patient }\end{array}$ & 9411 & 10149 & 9707 & 9850 \\
QALYs per patient & 1.461 & 1.442 & 1.438 & 1.418 \\
ICER & 12.623 & 12.675 & 12.683 & 12.769 \\
NHB & & & & \\
NMB & & & & \\
\hline
\end{tabular}

Abbreviations: USD, United states Dollars; QALYs, quality-adjusted life years; ICER, incremental costeffectiveness ratio; NHB, net health benefit; NMB, net monetary benefit; NT, no treatment; RW, real-world setting; FC, full compliance; FP, full persistence

Table 3 Results of one-way analyses

\begin{tabular}{|c|c|c|c|c|c|c|c|}
\hline \multirow[t]{2}{*}{ Parameter } & \multirow{2}{*}{$\begin{array}{l}\text { Cost (2018 } \\
\text { USD) } \\
\text { No } \\
\text { treatment }\end{array}$} & \multirow{2}{*}{$\begin{array}{l}\text { Cost (2018 } \\
\text { USD) } \\
\text { Alendronate }\end{array}$} & \multirow[t]{2}{*}{$C$} & \multicolumn{3}{|c|}{ Effectiveness(Effedtiss)eness(QALYs) } & \multirow[t]{2}{*}{$\begin{array}{l}\text { ICER(USD/ } \\
\text { QALY } \\
\text { gained) }\end{array}$} \\
\hline & & & & No treat & Alendrc & & \\
\hline $\begin{array}{l}\text { Starting } \\
\text { age of } \\
\text { treatment } \\
: 80\end{array}$ & 4816 & 5283 & 467 & 5.351 & 5.400 & 0.049 & 9530.612 \\
\hline $\begin{array}{l}\text { Starting } \\
\text { age of } \\
\text { treatment } \\
: 75\end{array}$ & 6483 & 7003 & 520 & 7.321 & 7.368 & 0.047 & 11063.830 \\
\hline $\begin{array}{l}\text { Starting } \\
\text { age of } \\
\text { treatment } \\
: 70\end{array}$ & 8076 & 8651 & 575 & 9.631 & 9.675 & 0.044 & 13068.182 \\
\hline $\begin{array}{l}5 \text {-year } \\
\text { time } \\
\text { horizon }\end{array}$ & 604 & 1598 & 994 & 3.860 & 3.870 & 0.010 & 99400.000 \\
\hline $\begin{array}{l}\text { No } \\
\text { residual } \\
\text { effect }\end{array}$ & 9422 & 10260 & 838 & 12.649 & 12.666 & 0.017 & 49294.118 \\
\hline $\begin{array}{l}\text { Discount } \\
\text { rate: } 0\end{array}$ & 10557 & 11421 & 864 & 13.867 & 13.941 & 0.074 & 11675.676 \\
\hline $\begin{array}{l}\text { Discount } \\
\text { rate: } 0.05\end{array}$ & 8407 & 9032 & 625 & 11.661 & 11.692 & 0.031 & 20161.290 \\
\hline $\begin{array}{l}\text { Fracture } \\
\text { costs } 30 \% \\
\text { higher }\end{array}$ & 12319 & 12808 & 489 & 12.654 & 12.681 & 0.027 & 18111.111 \\
\hline $\begin{array}{l}\text { Fracture } \\
\text { costs } 30 \% \\
\text { lower }\end{array}$ & 6605 & 7329 & 724 & 12.647 & 12.686 & 0.039 & 18564.103 \\
\hline
\end{tabular}




\begin{tabular}{|c|c|c|c|c|c|c|c|}
\hline \multirow{2}{*}{$\begin{array}{l}\text { Parameter } \\
\text { Fracture } \\
\text { disutilities } \\
30 \% \\
\text { higher }\end{array}$} & \multirow{2}{*}{$\begin{array}{l}\text { Cost (2018 } \\
\text { USD) } \\
9461\end{array}$} & \multirow{2}{*}{$\begin{array}{l}\text { Cost (2018 } \\
\text { USD) } \\
10098\end{array}$} & \multirow{2}{*}{$\begin{array}{l}C \\
637\end{array}$} & \multicolumn{3}{|c|}{ Effectiveness(QffelcYis)eness(QALYs) } & \multirow{2}{*}{$\begin{array}{l}\text { ICER(USD } \\
\text { QALY } \\
\text { gained) } \\
17216.216\end{array}$} \\
\hline & & & & 12.888 & 12.925 & 0.037 & \\
\hline $\begin{array}{l}\text { Fracture } \\
\text { disutilities } \\
30 \% \text { lower }\end{array}$ & 9542 & 10176 & 634 & 12.438 & 12.474 & 0.036 & 17611.111 \\
\hline $\begin{array}{l}\text { Alen costs } \\
30 \% \\
\text { higher }\end{array}$ & 9418 & 10204 & 786 & 12.634 & 12.678 & 0.044 & 17863.636 \\
\hline $\begin{array}{l}\text { Alen costs } \\
30 \% \text { lower }\end{array}$ & 9457 & 9925 & 468 & 12.641 & 12.679 & 0.038 & 12315.789 \\
\hline $\begin{array}{l}\text { Excess } \\
\text { mortality } \\
50 \% \\
\text { higher }\end{array}$ & 11647 & 12273 & 626 & 12.917 & 12.945 & 0.028 & 22357.143 \\
\hline $\begin{array}{l}\text { Excess } \\
\text { mortality } \\
0 \%\end{array}$ & 8819 & 9483 & 664 & 12.571 & 12.604 & 0.033 & 20121.212 \\
\hline
\end{tabular}

\section{Figure legends}

Fig.1. Results of probabilistic sensitivity analyses. The cost-effectiveness acceptability curves represent probabilities of being cost-effective achieved by the alendronate strategy compared to no treatment at willingness-to-pay thresholds for postmenopausal osteoporotic women.

Fig.2. Impact of medication compliance and persistence on therapy, disease, and total costs and on health outcomes (expressed as number of fractures prevented and QALY gained). QALY, quality-adjusted life-year.

Fig.3. Economic value of persistence-enhancing interventions according to a given range of their costs and effectiveness values. Each block represents a possible intervention characterized by its cost and effectiveness. The color coding denotes the cost-effectiveness of the intervention. 

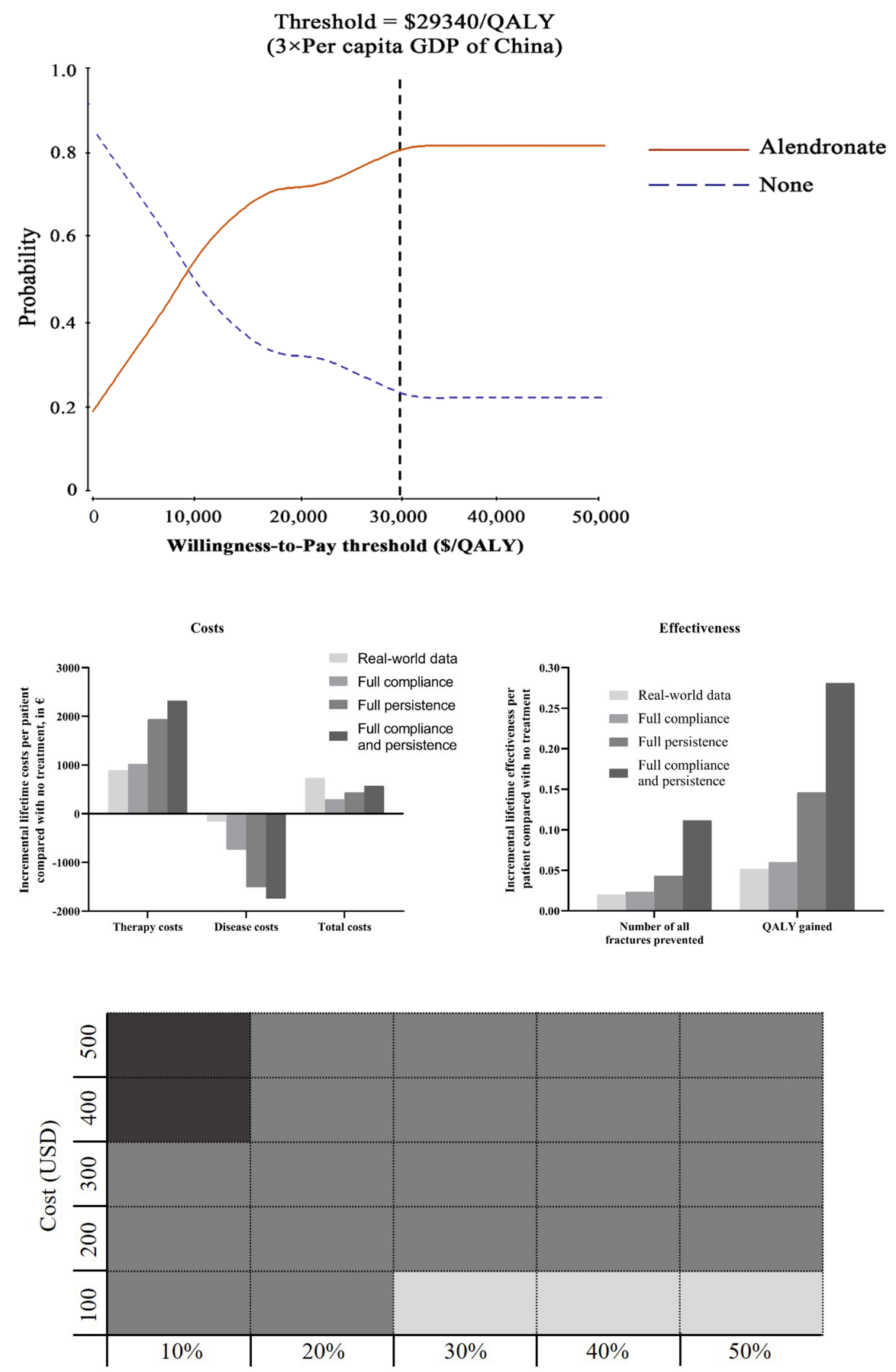

Effectiveness - Retative Reduction in Treatment Discontinuation 